\title{
Resonant excitation of the axion field during the QCD phase transition
}

\author{
Pierre Sikivie $\odot$ and Wei Xue \\ Department of Physics, University of Florida, Gainesville, Florida 32611, USA
}

(Received 25 October 2021; accepted 28 January 2022; published 23 February 2022)

\begin{abstract}
We find that the adiabatic fluctuations produced in the primordial plasma by cosmological inflation resonantly excite the axion field during the QCD phase transition by pumping axions from low momentum modes to modes with momentum up to of order $\sqrt{3} m$ where $m$ is the axion mass. We derive the momentum distribution of the excited axions. The fraction of cold axions that get excited is of order one if the axion mass is larger than a few $\mu \mathrm{eV}$. The effect occurs whether inflation happens before or after the Peccei-Quinn phase transition.
\end{abstract}

DOI: 10.1103/PhysRevD.105.043533

\section{INTRODUCTION}

The axion is a hypothetical particle postulated to solve the strong $C P$ problem, i.e., to explain why the strong interactions conserve the discrete symmetries $P$ and $C P$ in spite of the fact that these symmetries are broken in the Standard Model of particle physics as a whole [1-3]. Axions are independently motivated by cosmology because a population of cold axions is produced during the QCD phase transition, when the temperature is of order $1 \mathrm{GeV}\left(k_{B}=\hbar=c=1\right)$ [4]. In the simplest scenarios, axions account for the cold dark matter of the Universe if the axion mass is of order $10^{-5} \mathrm{eV}$, with large uncertainties [5].

The axion [2] is the quasi-Nambu-Golstone boson resulting from the spontaneous breaking of the $\mathrm{U}_{\mathrm{PQ}}(1)$ anomalous global symmetry that Peccei and Quinn [1] postulated. The properties of the axion depend for the most part on a single parameter $f_{a}$ called the axion decay constant. In particular the axion mass is

$$
m \simeq 6 \mu \mathrm{eV}\left(\frac{10^{12} \mathrm{GeV}}{f_{a}}\right)
$$

at temperatures below $100 \mathrm{MeV}$. At temperatures $T$ of order $1 \mathrm{GeV}$ and higher, the axion mass is estimated to be [4]

$$
m(T) \simeq 4 \times 10^{-9} \mathrm{eV}\left(\frac{10^{12} \mathrm{GeV}}{f_{a}}\right)\left(\frac{\mathrm{GeV}}{T}\right)^{4}
$$

Published by the American Physical Society under the terms of the Creative Commons Attribution 4.0 International license. Further distribution of this work must maintain attribution to the author(s) and the published article's title, journal citation, and DOI. Funded by SCOAP . using the dilute instanton gas approximation [6]. At intermediate temperatures, between $1 \mathrm{GeV}$ and $100 \mathrm{MeV}$, the axion mass may be estimated from QCD lattice simulations $[7,8]$. The precise manner in which the axion mass turns on during the QCD phase transition affects the quantitative results derived in the present paper.

An important source of uncertainty when estimating the abundance of cold axions, is whether the $\mathrm{U}_{\mathrm{PQ}}(1)$ symmetry is spontaneously broken before or after the epoch of inflationary expansion $[9,10]$ that homogenizes the very early universe. $\mathrm{U}_{\mathrm{PQ}}(1)$ becomes spontaneously broken when the temperature falls below some critical value of order $f_{a}$, during the so-called PQ phase transition. If this phase transition occurs before inflation, the axion field gets homogenized by the inflationary expansion, so that at the start of the QCD phase transition the axion field oscillations begin with almost the same amplitude everywhere. Unless this initial amplitude happens to be zero, which would be an unexpected accident, axions are produced in a process of "vacuum realignment" [4]. If the PQ phase transition occurs after inflation, the axion field enters the QCD phase transition with random initial amplitudes, uncorrelated from one QCD horizon volume to the next. In that case cold axions are produced by vacuum realignment and by the decay of axion strings and domain walls [5].

In this paper we are interested in the momentum spectrum of cold axions at the end of the QCD phase transition. If inflation occurs after the PQ phase transition the axion field is homogenized by the inflationary expansion. However, as is well known, the axion fluid has perturbations which originate as quantum mechanical fluctuations in the axion field during inflation [11]. These perturbations have been studied extensively because they produce isocurvature density perturbations that may be inconsistent with the observed cosmic microwave background (CMB) anisotropies. The observed anisotropies are 
consistent with purely adiabatic density perturbations. The absence of isocurvature perturbations qualitatively requires $H_{I} \lesssim 10^{-5} f_{a}$ where $H_{I}$ is the Hubble constant during inflation.

We are interested in length scales much smaller than those constrained by the CMB observations since the QCD horizon stretched to the present epoch is approximately $10^{17} \mathrm{~cm}$, much smaller than the length scales probed in the CMB. On the other hand, since the power spectrum of density perturbations predicted by inflation is very nearly flat, observations or theoretical considerations at one scale are potentially relevant to the other scales as well.

The quantum mechanical fluctuations in the axion field during inflation are not the only source of perturbations in the axion fluid in case inflation occurs after the PQ phase transition. A second source is the effect on the axion fluid of the adiabatic perturbations in the primordial plasma [12]. For the wave vectors of interest to us, this second source of axion fluid perturbations dominates. Indeed we find that the adiabatic perturbations in the primordial plasma resonantly excite the axion field. As far as we know, this effect has not been anticipated or commented on until now.

The resonant excitation can be thought of as the process $\tilde{\gamma}(\vec{k})+\phi(\overrightarrow{0}) \rightarrow \phi(\vec{k})$, where $\tilde{\gamma}(\vec{k})$ is a plasmon and $\phi(\vec{k})$ an axion, both with momentum $\vec{k}$. It is shown in Sec. IV that for every comoving wave vector $\vec{k}$ there is a time interval during which this process conserves energy exactly or sufficiently well that it occurs efficiently. During that time, axions are transferred from the very low momentum modes that they occupy initially to modes of momentum up to of order $\sqrt{3} \mathrm{~m}$. The process transfers energy from the plasma oscillations to the axion fluid. The process is qualitatively the same whether inflation occurs before or after the PQ phase transition because in either case the initial momenta of the axions are much less than the transferred momenta. However, it is possible to derive the momentum spectrum of cold axions with the greatest precision in case inflation occurs after the PQ phase transition, and this is one of our goals here. Our treatment is complete only for modes that enter the horizon well before the QCD phase transition. The analysis of modes that enter the horizon near the start of and during the QCD phase transition is left for future work.

The outline of our paper is as follows. In Sec. II, we state the equation of motion for the axion field, the spectrum of initial perturbations in the axion field, and the spectrum of adiabatic perturbations in the primordial plasma. In Sec. III we evolve the initial perturbations in the axion field and derive the resulting axion momentum spectrum. In Sec. IV we derive the perturbations in the axion field induced by the adiabatic fluctuations in the primoridial plasma and the resulting axion momentum spectrum. Section V summarizes our results.

\section{EQUATION OF MOTION AND INITIAL CONDITIONS}

Ignoring $\lambda \phi^{4}$ self-interactions, the axion field $\phi(\vec{x}, t)$ satisfies a linear equation of motion:

$$
D^{\mu} \partial_{\mu} \phi(\vec{x}, t)-m^{2}(T(\vec{x}, t)) \phi(\vec{x}, t)=0
$$

where $m(T)$ is the temperature-dependent axion mass. We are interested in scalar perturbations only. So we may adopt the so-called conformal-Newtonian gauge for the spacetime metric:

$$
\begin{aligned}
g_{\alpha \beta}(x) d x^{\alpha} d x^{\beta}= & -(1+2 \Psi(\vec{x}, t)) d t^{2} \\
& +a^{2}(t)(1+2 \Phi(\vec{x}, t)) d \vec{x} \cdot d \vec{x} .
\end{aligned}
$$

During the QCD phase transition, ignoring small corrections due to changes in the number of thermal degrees of freedom as a function of temperature, the scale factor $a(t) \propto \sqrt{t}$, and the Hubble expansion rate $H(t)=\frac{1}{2 t}$. When only zeroth and first order terms in $\Psi(\vec{x}, t), \Phi(\vec{x}, t)$ and the temperature fluctuations $\delta T(\vec{x}, t)$ are included, Eq. (2.1) becomes

$$
\begin{aligned}
& (-1+2 \Psi) \partial_{t} \partial_{t} \phi+\partial_{t} \Psi \partial_{t} \phi+\frac{1}{a^{2}} \partial_{j} \Psi \partial_{j} \phi+(1-2 \Phi)\left(\frac{1}{a^{2}} \partial_{j} \partial_{j} \phi-3 H \partial_{t} \phi\right)-3\left(\partial_{t} \Phi+2 H \Phi-2 H \Psi\right) \partial_{t} \phi \\
& +\frac{1}{a^{2}} \partial_{j} \Phi \partial_{j} \phi-\left[m^{2}(T(t))+\frac{d m^{2}}{d T} \delta T\right] \phi=0
\end{aligned}
$$

where $j=1,2,3$ labels the space directions.

At temperatures of order $1 \mathrm{GeV}$, the axion mass is given by Eq. (1.2). It implies the time dependence:

$$
m(t) \simeq 0.9 \times 10^{6} \frac{1}{\mathrm{sec}}\left(\frac{t}{10^{-7} \mathrm{sec}}\right)^{2}\left(\frac{10^{12} \mathrm{GeV}}{f_{a}}\right) .
$$

We define $t_{1}$ such that $m\left(t_{1}\right) t_{1}=1$. Eq. (2.4) implies

$$
t_{1} \simeq 2 \times 10^{-7} \sec \left(\frac{f_{a}}{10^{12} \mathrm{GeV}}\right)^{\frac{1}{3}}
$$

at which time the temperature is 


$$
T_{1} \simeq 1 \mathrm{GeV}\left(\frac{10^{12} \mathrm{GeV}}{f_{a}}\right)^{\frac{1}{6}}
$$

At lower temperatures, the axion mass may be obtained by QCD lattice simulations. The results of Sz. Borsanyi et al. [7] and C. Bonati et al. [8] are consistent with one another and with the temperature dependence given in Eq. (1.2) down to $T_{2} \simeq 160 \mathrm{MeV}$, where $m(T)$ turns over and becomes its zero temperature value given in Eq. (1.1). The age of the universe at temperature $T_{2}$ is $2 \times 10^{-5} \mathrm{sec}$ [13] when the thinning out of thermal degrees of freedom during the QCD phase transition is taken into account. In our estimates below, to simplify the calculations somewhat, we will ignore the thinning out of thermal degrees of freedom and assume

$$
\begin{aligned}
m(t) & =\frac{1}{t_{1}}\left(\frac{t}{t_{1}}\right)^{2}=m\left(\frac{t}{t_{2}}\right)^{2} & & \text { for } t<t_{2} \\
& =m & & \text { for } t>t_{2} .
\end{aligned}
$$

This requires $t_{2}=\sqrt{m t_{1}^{3}} \simeq 10^{-5} \mathrm{sec}$. In principle, our approach can be used for any smooth turn-on of the axion mass. It fails if the axion mass has a discontinuity as a function of temperature, as may occur if the QCD phase transition is first order.

The zeroth order equation for the axion field is

$$
\left(\frac{d^{2}}{d t^{2}}+\frac{3}{2 t} \frac{d}{d t}+m^{2}(t)\right) \phi^{(0)}(t)=0
$$

For $t \ll t_{1}$, when the axion mass is negligible, Eq. (2.8) is solved by

$$
\phi^{(0)}(t)=A+D \frac{1}{\sqrt{t}}
$$

where $A$ and $D$ are constants. We set $D=0$ since $\frac{d}{d t} \phi^{(0)}=$ 0 at the end of inflation. For $t \gg t_{1}$, the change in the axion mass is adiabatic $\left(\frac{d m}{d t} \ll m^{2}\right)$ so that

$\phi^{(0)}(t)=\frac{B}{\sqrt{t_{1} m(t)}}\left(\frac{t_{1}}{t}\right)^{\frac{3}{4}} \cos \left(\int_{0}^{t} d t^{\prime} m\left(t^{\prime}\right)+\delta\right)$.

The amplitude $B$ and phase $\delta$ can be obtained in terms of $A$ by solving Eq. (2.8) numerically. Figure 1 shows the function $\phi^{(0)}(t)$ for $A=1$. After the QCD phase transition, the energy density in the zero mode $\phi^{(0)}$ is

$$
\rho^{(0)}(t)=\frac{B^{2}}{2 t_{1}}\left(\frac{t_{1}}{t}\right)^{\frac{3}{2}} m
$$

which is indeed the standard result [4].

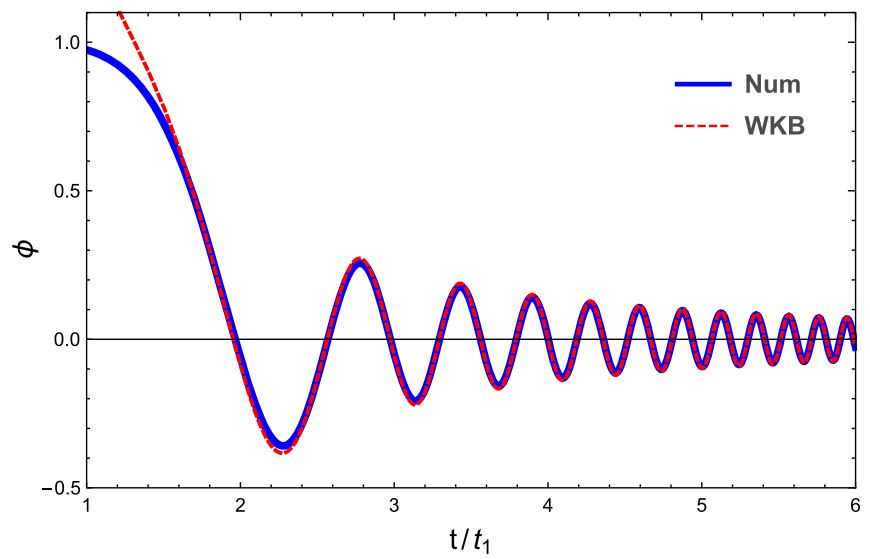

FIG. 1. Plot of the axion field zero mode $\phi^{(0)}(t)$ as it begins to oscillate at the onset of the QCD phase transition. The continuous line is the result of numerically integrating Eq. (2.8) starting with an initial constant value. The dashed line is the time dependence given in Eq. (2.10) for an appropriate choice of the phase $\delta$ and amplitude $B$.

The first order equation for the perturbations $\phi^{(1)}(\vec{x}, t)=$ $\phi(\vec{x}, t)-\phi^{(0)}(t)$ in the axion field is

$$
\begin{aligned}
& \partial_{t}^{2} \phi^{(1)}(\vec{k}, t)+\frac{3}{2 t} \phi^{(1)}(\vec{k}, t) \\
& \quad+\left(\frac{\vec{k} \cdot \vec{k}}{a^{2}(t)}+m^{2}(t)\right) \phi^{(1)}(\vec{k}, t) \\
& =j(\vec{k}, t)
\end{aligned}
$$

where

$$
\begin{aligned}
j(\vec{k}, t)= & 2 \Psi(\vec{k}, t) \partial_{t}^{2} \phi^{(0)}(t)+\left(\partial_{t} \Psi(\vec{k}, t)-3 \partial_{t} \Phi(\vec{k}, t)\right. \\
& +6 H(t) \Psi(\vec{k}, t)) \partial_{t} \phi^{(0)}(t)-\frac{d m^{2}}{d T}(T(t)) \delta T(\vec{k}, t) \phi^{(0)}(t) .
\end{aligned}
$$

The $\phi^{(1)}(\vec{k}, t)$ are the Fourier modes of $\phi^{(1)}(\vec{x}, t)$, defined by

$$
\phi^{(1)}(\vec{x}, t)=\int \frac{d^{3} k}{(2 \pi)^{3}} \phi^{(1)}(\vec{k}, t) e^{i \vec{k} \cdot \vec{x}}
$$

$\Psi(\vec{k}, t), \Phi(\vec{k}, t)$, and $\delta T(\vec{k}, t)$ are likewise the Fourier modes of $\Psi(\vec{x}, t), \Phi(\vec{x}, t)$, and $\delta T(\vec{x}, t)$.

The quantum mechanical fluctuations in the inflaton field produce adiabatic fluctuations. Provided anisotropic stresses in the primordial plasma are small, as is normally the case, $\Psi(\vec{k}, t)=-\Phi(\vec{k}, t)$ [14]. We will assume this to be true henceforth. The power spectrum of $\Phi$ fluctuations when they reenter the horizon $(\mathrm{k}=\mathrm{a}(\mathrm{t}) \mathrm{H}(\mathrm{t}))$ is 


$$
\begin{aligned}
\left.\left\langle\Phi(\vec{k}, t) \Phi^{*}\left(\vec{k}^{\prime}, t\right)\right\rangle\right|_{k=a H} & \equiv\left\langle\Phi_{p}(\vec{k}) \Phi_{p}^{*}\left(\vec{k}^{\prime}\right)\right\rangle \\
& =(2 \pi)^{3} \delta\left(\vec{k}-\vec{k}^{\prime}\right) \frac{8 \pi G}{9 k^{3}} \frac{H_{I}^{2}}{\epsilon}
\end{aligned}
$$

where $G$ is Newton's gravitational constant, $H_{I}$ is the expansion rate during inflation, and $\epsilon \equiv \frac{d}{d t} \frac{1}{H_{I}(t)}$. The amplitude of adiabatic perturbations is derived from observations of the cosmic microwave background anisotropies on scales of order the present horizon:

$$
\frac{8 \pi G}{9 k^{3}} \frac{H_{I}^{2}}{\epsilon}=\frac{50 \pi^{2}}{9 k^{3}}\left(\frac{k}{H_{0}}\right)^{n-1} \delta_{H}^{2}\left(\frac{\Omega_{m}}{D_{1}\left(t_{0}\right)}\right)^{2}
$$

where $H_{0}$ is the present expansion rate, $n \simeq 0.96$ is the scalar power index, $\delta_{H} \simeq 4.6 \times 10^{-5}$ is the amplitude of adiabatic density perturbations when they reenter the horizon, $\Omega_{m} \simeq 0.3$ is the fraction of the critical energy density in matter today, and $D_{1}\left(t_{0}\right) \simeq 0.8$ is the growth function. Equation (2.16) and the various parameter estimates are taken from Ref. [14]. Equation (2.16) applies on wave vector scales of order $H_{0}^{-1}$. We extrapolate it, over approximately 13 orders of magnitude, to modes that enter the horizon shortly before the QCD phase transition. This yields

$$
\frac{8 \pi G H_{I}^{2}}{9 \epsilon} \equiv C \simeq 4.9 \times 10^{-9} .
$$

Our results in Sec. IV are expressed in terms of $C$, with the value given in Eq. (2.17) substituted only at the end to make final estimates, in case the assumptions made above about the inflationary model need to be modified.

After they have entered the horizon, the $\Phi$ fluctuations oscillate as follows [14]

$$
\begin{aligned}
\Phi(\vec{k}, t) & =3 \Phi_{p}(\vec{k})\left(\frac{\sqrt{3}}{2 k \sqrt{t t_{1}}}\right)^{3}\left[\sin \left(2 k \sqrt{\frac{t t_{1}}{3}}\right)-2 k \sqrt{\frac{t t_{1}}{3}} \cos \left(2 k \sqrt{\frac{t t_{1}}{3}}\right)\right] \\
& =-\frac{9}{4 k^{2} t t_{1}} \Phi_{p}(\vec{k}) \cos \left(2 k \sqrt{\frac{t t_{1}}{3}}\right) \quad \text { for } k \sqrt{t t_{1}} \gg 1 .
\end{aligned}
$$

We have normalized the scale factor so that $a\left(t_{1}\right)=1 . \vec{k}$ is thus the physical wavevector at time $t_{1}$. The $\Phi$ fluctuations are accompanied by fluctuations in the temperature [14]

$$
\begin{aligned}
\frac{\delta T(\vec{k}, t)}{T(t)} & =\left(\frac{1}{2}+\frac{2 k^{2} t t_{1}}{3}\right) \Phi(\vec{k}, t)+\frac{t}{4} \frac{d}{d t} \Phi(\vec{k}, t) \\
& =-\frac{3}{2} \Phi_{p}(\vec{k}) \cos \left(2 k \sqrt{\frac{t t_{1}}{3}}\right) \quad \text { for } k \sqrt{t t_{1}} \gg 1
\end{aligned}
$$

The quantum mechanical fluctuations in the axion field, generated during inflation, have power spectrum

$$
\begin{aligned}
\left.\left\langle\delta \phi(\vec{k}, t) \delta \phi^{*}\left(\vec{k}^{\prime}, t\right)\right\rangle\right|_{k=a H} & \equiv\left\langle\phi_{p}(\vec{k}) \phi_{p}^{*}\left(\vec{k}^{\prime}\right)\right\rangle \\
& =(2 \pi)^{3} \delta\left(\vec{k}-\vec{k}^{\prime}\right) \frac{H_{I}^{2}}{2 k^{3}}
\end{aligned}
$$

when they reenter the horizon.

There are therefore two kinds of perturbations in the axion field

$$
\phi(\vec{x}, t)=\phi^{(0)}(t)+\phi_{\delta \phi}^{(1)}(\vec{x}, t)+\phi_{\Phi}^{(1)}(\vec{x}, t)
$$

where the $\phi_{\delta \phi}^{(1)}$ perturbations originate as quantum mechanical fluctuations in the axion field during inflation and the $\phi_{\Phi}^{(1)}$ perturbations are induced in the axion field by the adiabatic fluctuations in the primordial plasma. The two kinds of perturbations are assumed to be uncorrelated, i.e.,

$$
\left\langle\Phi_{p}(\vec{k}) \phi_{p}\left(\vec{k}^{\prime}\right)\right\rangle=0 .
$$

The fluid density in nonzero mode axions is then a simple sum:

$$
\rho^{(1)}=\rho_{\delta \phi}^{(1)}+\rho_{\Phi}^{(1)}
$$

where $\rho_{\delta \phi}^{(1)}$ and $\rho_{\Phi}^{(1)}$ are the contributions from $\phi_{\delta \phi}^{(1)}$ and $\phi_{\Phi}^{(1)}$ respectively. They are evaluated in Secs. III and IV respectively.

\section{QUANTUM-MECHANICAL FLUCTUATIONS OF THE AXION FIELD}

In this section we set $j(\vec{x}, t)=0$ and derive how the axion field perturbations evolve in the absence of adiabatic perturbations in the primordial plasma. The equation of motion is

$$
\left[\partial_{t}^{2}+\frac{3}{2 t} \partial_{t}+k^{2} \frac{t_{1}}{t}+m^{2}(T(t))\right] \phi^{(1)}(\vec{k}, t)=0 .
$$

It may also be written 


$$
\left(\partial_{t}^{2}+\Omega^{2}(k, t)\right) B^{(1)}(\vec{k}, t)=0
$$

where $B^{(1)}(\vec{k}, t)$ is defined by

$$
\phi^{(1)}(\vec{k}, t)=\left(\frac{t_{1}}{t}\right)^{\frac{3}{4}} B^{(1)}(\vec{k}, t)
$$

and

$$
\Omega(k, t)=\sqrt{\frac{3}{16 t^{2}}+k^{2} \frac{t_{1}}{t}+m^{2}(t)} .
$$

For $t \ll t_{1}$, when the axion mass is negligible, Eq. (3.1) is solved exactly by

$$
\phi^{(1)}(\vec{k}, t)=\phi_{p}(\vec{k}) \frac{1}{2 k \sqrt{t t_{1}}} \sin \left(2 k \sqrt{t t_{1}}\right)
$$

$\phi_{p}(\vec{k})$ being the initial value of the perturbation.

For $k \gg \frac{1}{t_{1}}$ the turn on of the axion mass is adiabatic and therefore

$\phi^{(1)}(\vec{k}, t)=\frac{C(\vec{k})}{\sqrt{\omega(k, t)}}\left(\frac{t_{1}}{t}\right)^{\frac{3}{4}} \sin \left(\int_{0}^{t} d t^{\prime} \omega\left(k, t^{\prime}\right)\right)$

solves Eq. (3.1) for $t \gtrsim t_{1}$ with

$$
\omega(k, t)=\sqrt{k^{2} \frac{t_{1}}{t}+m^{2}(t)} .
$$

The right-hand side (rhs) of Eq. (3.5) equals that of Eq. (3.6) for $t \ll t_{1}$ provided $C(\vec{k})=\phi_{p}(\vec{k}) /\left(2 \sqrt{k} t_{1}\right)$. We have therefore

$\phi^{(1)}(\vec{k}, t)=\frac{\phi_{p}(\vec{k})}{2 t_{1} \sqrt{k \omega(k, t)}}\left(\frac{t_{1}}{t}\right)^{\frac{3}{4}} \sin \left(\int_{0}^{t} d t^{\prime} \omega\left(k, t^{\prime}\right)\right)$

for all $t$ when $j(\vec{k}, t)=0$ and $k \gg \frac{1}{t_{1}}$. Equation (3.8) was compared with the result of numerically solving Eq. (3.1), and found to be practically indistinguishable from it for $k \geq 2 / t_{1}$. Figure 2 shows the comparison for $k=2 / t_{1}$.

Equation (3.8) implies for $t \gg t_{1}$

$$
\begin{aligned}
\rho_{\delta \phi}^{(1)} & =\left(\frac{t_{1}}{t}\right)^{\frac{3}{2}} \int \frac{d^{3} k}{(2 \pi)^{3}} \int \frac{d^{3} k^{\prime}}{(2 \pi)^{3}}\left\langle\phi_{p}(\vec{k}) \phi_{p}\left(\vec{k}^{\prime}\right)\right\rangle \frac{m}{8 t_{1}^{2} k} \\
& \simeq m\left(\frac{t_{1}}{t}\right)^{\frac{3}{2}} \frac{H_{I}^{2}}{16 t_{1}^{2}} \int_{\frac{1}{t_{1}}} \frac{d^{3} k}{(2 \pi)^{3}} \frac{1}{k^{4}} \\
& \simeq m\left(\frac{t_{1}}{t}\right)^{\frac{3}{2}}\left(\frac{H_{I}}{2 \pi}\right)^{2} \frac{1}{8 t_{1}}
\end{aligned}
$$

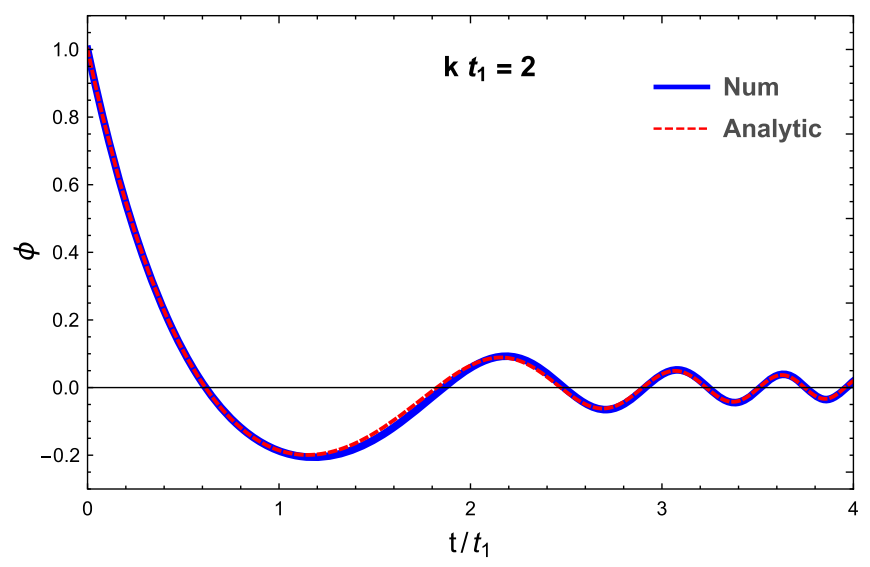

FIG. 2. Plot of $\phi^{(1)}(\vec{k}, t)$ for $k=2 / t_{1}$. The continuous line is the result of numerically solving Eq. (3.1). The dashed line is the time dependence given in Eq. (3.8).

including the contribution of all modes that enter the horizon before $t_{1} \cdot \rho_{\delta \phi}^{(1)}$ is very small compared to $\rho^{(0)}$ since the observed absence of isocurvature perturbations implies $H_{I} \lesssim 10^{-5} f_{a}$.

\section{RESONANT EXCITATION OF THE AXION FIELD}

We now turn to the effects that result from the adiabatic fluctuations in the plasma. Fig. 3 summarizes our conclusions. It shows the spectrum of excited axions. The horizontal axis is divided into three regions. The region $k_{*}<k<k_{c}$ is dominated by axions which are resonantly excited at a time intermediate between $t_{1}$ and $t_{2}$. The region $k<k_{*}$ is dominated by axions which are excited by a near resonance effect which occurs from the time they are

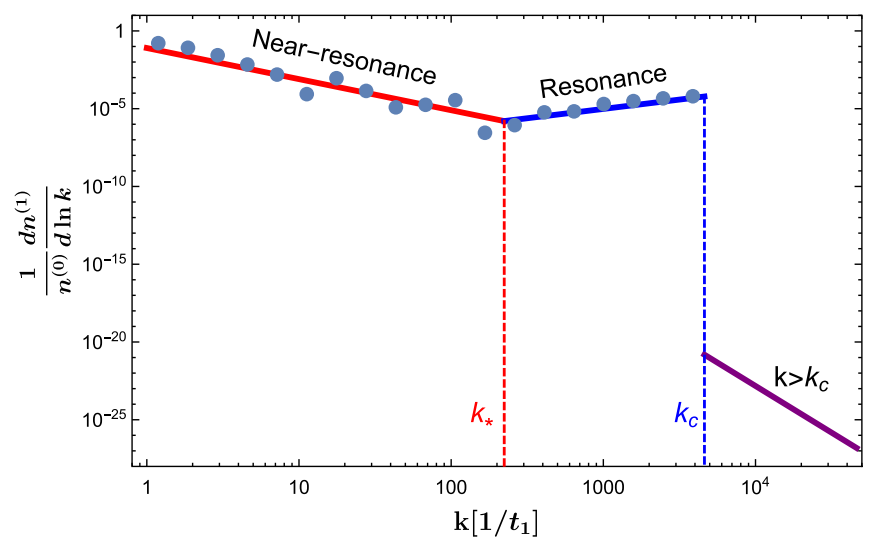

FIG. 3. Spectrum of axions excited by the resonance proper (blue and purple lines) and near resonance effects (red line), for $m=1 \mu \mathrm{eV}$. Only the dominant contribution is shown for given $k$. The contribution to the axion spectrum from the quantum mechanical fluctuations in the axion field, discussed in Sec. III, is not shown here. The grey dots indicate the results from numerically solving Eq. (4.3). 
resonantly excited till time $t_{2}$. The axions that are resonantly excited after time $t_{2}$ have wave vectors $k>k_{c}$.

\section{A. General remarks}

Equation (2.12) shows that $j(\vec{k}, t)$ acts as a source for $\phi^{(1)}(\vec{k}, t)$. This source is turned off for $t \ll t_{1}$, because $\partial_{t} \phi^{(0)}$ and $\frac{\partial m^{2}}{\partial T}$ are negligible then. We rewrite Eqs. (2.12) and (2.13) in terms of $B^{(0)}(t)$ defined by

$$
\phi^{(0)}(t)=B^{(0)}(t)\left(\frac{t_{1}}{t}\right)^{\frac{3}{4}}
$$

and $B^{(1)}(\vec{k}, t)$ defined by Eq. (3.3), and use Eq. (2.8) to eliminate $\partial_{t}^{2} \phi^{(0)}$. This yields

$$
\left(\partial_{t}^{2}+\Omega^{2}(k, t)\right) B^{(1)}(\vec{k}, t)=-4 \partial_{t} \Phi(\vec{k}, t) \partial_{t} B^{(0)}(t)+\left[-\frac{d m^{2}}{d T} \delta T(\vec{k}, t)+2 m^{2}(t) \Phi(\vec{k}, t)+6 H(t) \partial_{t} \Phi(\vec{k}, t)\right] B^{(0)}(t) .
$$

We substitute the late time $\left(k \sqrt{t t_{1}} \gg 1\right)$ behaviors of $\Phi(\vec{k}, t)$ and $\delta T(\vec{k}, t)$, given in Eqs. (2.18) and (2.19), and that of $B^{(0)}(t)$ implied by Eq. (2.10). This yields

$$
\left(\partial_{t}^{2}+\omega^{2}(k, t)\right) B^{(1)}(\vec{k}, t)=f(\vec{k}, t)
$$

with

$$
\begin{aligned}
f(\vec{k}, t)= & \frac{\Phi_{p}(\vec{k}) B}{\sqrt{t_{1} m(t)}}\left[\left(\frac{3}{2} \frac{d m^{2}}{d T}(t) T(t)-\frac{9 m^{2}(t)}{2 k^{2} t t_{1}}\right) \cos \left(\int_{0}^{t} d t^{\prime} m\left(t^{\prime}\right)+\delta\right) \cos \left(2 k \sqrt{\frac{t t_{1}}{3}}\right)\right. \\
& \left.+\frac{9 m(t)}{k t \sqrt{3 t t_{1}}} \sin \left(\int_{0}^{t} d t^{\prime} m\left(t^{\prime}\right)+\delta\right) \sin \left(2 k \sqrt{\frac{t t_{1}}{3}}\right)\right]
\end{aligned}
$$

for $k \sqrt{t t_{1}} \gg 1$.

Resonant excitation of the axion field occurs because $f(\vec{k}, t)$ includes terms that oscillate briefly with frequency $\omega(k, t)$. Indeed the frequencies that appear in $f(\vec{k}, t)$ are $\pm m(t) \pm \frac{k}{\sqrt{3}} \sqrt{\frac{t_{1}}{t}}$. Resonance occurs when

$$
\omega(k, t)=\sqrt{m^{2}(t)+k^{2} \frac{t_{1}}{t}}=m(t)+\frac{k}{\sqrt{3}} \sqrt{\frac{t_{1}}{t}},
$$

i.e., at time $t_{k}$ such that

$$
m\left(t_{k}\right)=\frac{k}{\sqrt{3}} \sqrt{\frac{t_{1}}{t_{k}}} .
$$

Henceforth we assume a specific time dependence for the axion mass: Eq. (2.4) for $t<t_{2}$ and Eq. (1.1) for $t>t_{2}$. Let us call $k_{c}$ the comoving wave vector magnitude of modes that are resonantly excited at time $t_{2}$. Equation (4.6) with $t_{k}=t_{2}$ and $m\left(t_{k}\right)=m$ implies

$$
k_{c}=m \sqrt{\frac{3 t_{2}}{t_{1}}} .
$$

Modes with comoving wave vector magnitude $k_{c}$ have physical wave vector magnitude equal to $\sqrt{3} m$ at time $t_{2}$. Modes with $k>k_{c}$ are resonantly excited at time

$$
t_{k}=\left(\frac{k}{k_{c}}\right)^{2} t_{2}
$$

which is after $t_{2}$. Those modes are discussed in Subsection IV. D. Modes with $k<k_{c}$ are resonantly excited at time

$$
t_{k}=\left(\frac{k}{k_{c}}\right)^{\frac{2}{5}} t_{2}
$$

which is before $t_{2}$, when the axion mass is still turning on. (Note that the larger its comoving wave vector magnitude $k$, the earlier a mode enters the horizon, but the later it gets resonantly excited.) The frequencies $\omega(k, t)$ and $\omega_{ \pm}(k, t) \equiv$ $m(t) \pm \frac{k}{\sqrt{3}} \sqrt{\frac{t_{1}}{t}}$ are plotted in Fig. 4 for $k=100 / t_{1}$. The figure shows that, after they are equal at time $t_{k}, \omega(k, t)$ and $\omega_{+}(k, t)$ stay close to each other. $\omega_{-}(k, t)$ soon comes close $\omega(k, t)$ as well. A near resonance excitation of the axion field occurs from approximately time $t_{k}$ till $t_{2}$, as discussed in Sec. IV C. The resonance proper near time $t_{k}$ is discussed in Sec. IV B.

We write

$$
B^{(1)}(\vec{k}, t)=\Phi_{p}(\vec{k})(a(k, t) \mathcal{B}(k, t)+\text { c.c. })
$$

where 


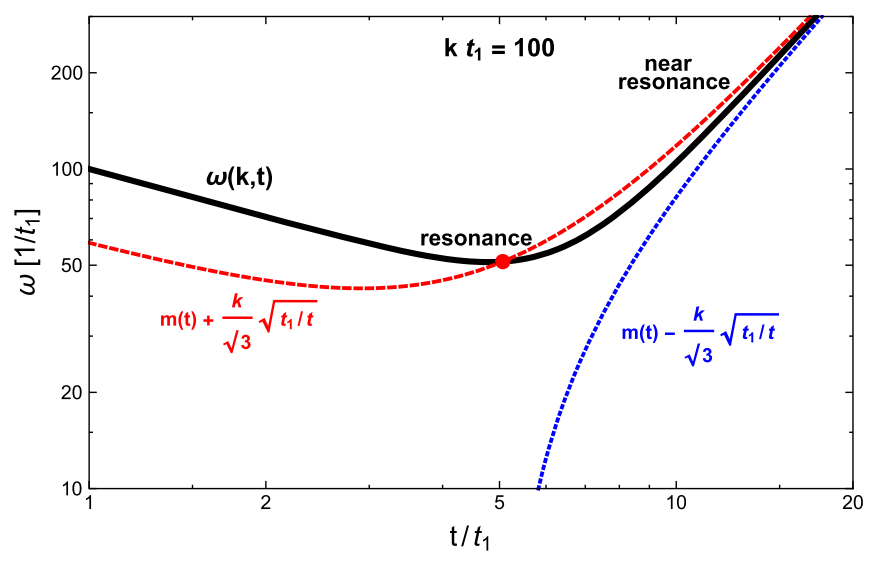

FIG. 4. Plot of the frequencies $\omega(k, t)$ and $\omega_{ \pm}(k, t)=m(t) \pm$ $\frac{k}{\sqrt{3}} \sqrt{\frac{t_{1}}{t}}$ as a function of time for $k=100 / t_{1}$.

$$
\mathcal{B}(k, t)=\frac{1}{\sqrt{\omega(k, t)}} e^{i \int_{0}^{t} d t^{\prime} \omega\left(k, t^{\prime}\right)}
$$

solves the equation of motion in the absence of source. We substitute Eq. (4.10) into Eq. (4.3), neglect $\frac{d^{2} a}{d t^{2}}$ relative to $\omega \frac{d a}{d t}$, and keep only terms in $f(\vec{k}, t)$ that produce resonance or near resonance. This yields

$$
\begin{aligned}
\frac{d}{d t} a(k, t) & =\frac{B}{2 i \sqrt{\omega(k, t)}}\left[g_{+}(k, t) e^{i\left(h_{+}(k, t)+\delta\right)}\right. \\
& \left.+g_{-}(k, t) e^{i\left(h_{-}(k, t)+\delta\right)}\right]
\end{aligned}
$$

where

$h_{ \pm}(k, t)=\int_{0}^{t} d t^{\prime}\left[ \pm \frac{k}{\sqrt{3}} \sqrt{\frac{t_{1}}{t^{\prime}}}+m\left(t^{\prime}\right)-\omega\left(k, t^{\prime}\right)\right]$

and

$g_{ \pm}(k, t)=\frac{1}{4 \sqrt{t_{1} m(t)}}\left[\frac{3 d m^{2}}{2} \frac{d T}{d T}(t) T(t)-\frac{9 m^{2}(t)}{2 k^{2} t_{1} t} \mp \frac{9 m(t)}{k t \sqrt{3 t_{1} t}}\right]$

The initial value of $\Phi_{p}(\vec{k}) a(k, t)$ is $\frac{\phi_{p}(\vec{k})}{4 i t_{1} \sqrt{k}}$ since the initial value of $\phi^{(1)}(\vec{k}, t)$ is $\phi_{p}(\vec{k})$; see Eq. (3.8). Its final value is therefore

$$
\Phi_{p}(\vec{k}) a(k)=\frac{\phi_{p}(\vec{k})}{4 i t_{1} \sqrt{k}}+\frac{\Phi_{p}(\vec{k}) B}{2 i}\left(I_{+}(k)+I_{-}(k)\right)
$$

with

$$
I_{ \pm}(k)=\int_{0}^{\infty} d t \frac{g_{ \pm}(k, t)}{\sqrt{\omega(k, t)}} e^{i\left(h_{ \pm}(k, t)+\delta\right)}
$$

Combining Eqs. (3.3), (4.10), (4.11), and (4.15)-(4.16), we obtain

$$
\begin{aligned}
\phi^{(1)}(\vec{k}, t)= & \frac{1}{\sqrt{m}}\left(\frac{t_{1}}{t}\right)^{\frac{3}{4}}\left[\frac{\phi_{p}(\vec{k})}{2 t_{1} \sqrt{k}} \sin (m t+\delta(k, t))\right. \\
& \left.+|I(k)| \Phi_{p}(\vec{k}) B \sin \left(m t+\delta^{\prime}(k, t)\right)\right]
\end{aligned}
$$

for $t \gg t_{2}$, where $I(k)=I_{+}(k)+I_{-}(k)$,

$$
\delta(k, t)=\int_{0}^{t} d t^{\prime} \sqrt{k^{2} \frac{t_{1}}{t^{\prime}}+m^{2}\left(t^{\prime}\right)}-m t
$$

and

$$
\delta^{\prime}(k, t)=\delta(k, t)+\arg (I(k))
$$

$\delta(k, t)$ and $\delta^{\prime}(k, t)$ vary only logarithmically with time after $t_{2}$.

Equations (4.17), (2.15), (2.17), (2.20), and (2.22) imply that the fluid density in nonzero mode axions is for $t \gg t_{2}$

$$
\rho^{(1)}=m^{2}\left\langle\left(\phi^{(1)}(\vec{x}, t)\right)^{2}\right\rangle=\rho_{\delta \phi}^{(1)}(t)+\rho_{\Phi}^{(1)}(t)
$$

with $\rho_{\delta \phi}^{(1)}$ given by Eq. (3.9) and

$$
\rho_{\Phi}^{(1)}(t)=m\left(\frac{t_{1}}{t}\right)^{\frac{3}{2}} \frac{C B^{2}}{2} \int \frac{d^{3} k}{(2 \pi)^{3}} \frac{1}{k^{3}}|I(k)|^{2}
$$

We may rewrite this as

$$
\rho_{\Phi}^{(1)}(t)=m \int d k \frac{d n_{\Phi}^{(1)}}{d k}(k, t)
$$

where

$$
\frac{d n_{\Phi}^{(1)}}{d k}(k, t)=\frac{C B^{2}}{4 \pi^{2} k}|I(k)|^{2}\left(\frac{t_{1}}{t}\right)^{\frac{3}{2}}
$$

is the number density of excited quanta per unit comoving wave vector magnitude.

\section{B. Resonant excitation for $\boldsymbol{k}<\boldsymbol{k}_{\boldsymbol{c}}$}

Modes with $k<k_{c}$ are resonantly excited at time $t_{k}$ given in Eq. (4.9). The resonance proper appears only in $I_{+}(k)$. We evaluate that integral using the saddle point approximation. Near time $t_{k}$ 


$$
\begin{aligned}
h_{+}(k, t) & =h_{+}\left(k, t_{k}\right)+\frac{1}{2} \frac{d^{2} h_{+}}{d t^{2}}\left(t_{k}\right)\left(t-t_{k}\right)^{2}+\cdots \\
& =h_{+}\left(k, t_{k}\right)+\frac{1}{4}\left(\frac{d m}{d t}\left(k, t_{k}\right)+\frac{m\left(t_{k}\right)}{2 t_{k}}\right)\left(t-t_{k}\right)^{2}+\cdots
\end{aligned}
$$

Neglecting the higher order terms in Eq. (4.24) and the slow variation of $g_{+}(k, t)$ and $\omega(k, t)$ inside the integral for $I_{+}(k)$ in Eq. (4.16), we obtain

$I_{r}(k) \simeq \frac{2 \sqrt{\pi} g_{+}\left(k, t_{k}\right)}{\sqrt{\omega\left(k, t_{k}\right)\left(\frac{d m}{d t}\left(t_{k}\right)+\frac{m\left(t_{k}\right)}{2 t_{k}}\right)}} e^{i\left(h_{+}\left(k, t_{k}\right)+\frac{\pi}{4}+\delta\right)}$

for the contribution to $I(k)$ from the resonance proper. The resonance proper occurs over an interval of time

$$
\Delta t_{k}=\frac{1}{\sqrt{\frac{d^{2} h_{+}}{d t^{2}}\left(k, t_{k}\right)}}=\frac{2}{\sqrt{5}} \sqrt{\frac{t_{2}}{m}}\left(\frac{k_{c}}{k}\right)^{\frac{1}{5}} .
$$

For $k<k_{c}$, we have

$g_{+}\left(k, t_{k}\right)=-\frac{1}{4 \sqrt{t_{1} m}}\left[12 m^{2}\left(\frac{k}{k_{c}}\right)^{\frac{6}{5}}+\frac{9}{2 t_{2}^{2}}\left(\frac{k_{c}}{k}\right)^{\frac{6}{5}}\right]$

and

$$
\omega\left(k, t_{k}\right)\left(\frac{d m}{d t}\left(t_{k}\right)+\frac{m\left(t_{k}\right)}{2 t_{k}}\right)=\frac{5 m^{2}}{t_{2}}\left(\frac{k}{k_{c}}\right)^{\frac{6}{5}} .
$$

Hence the contribution of the resonance proper to the spectrum of excited axions is

$\frac{d n_{r}^{(1)}}{d k}(k, t) \simeq \frac{9 C B^{2}}{5 \pi t_{1} k} m t_{2}\left(\frac{k}{k_{c}}\right)^{\frac{6}{5}}\left(1+\frac{3}{8\left(t_{2} m\right)^{2}}\left(\frac{k_{c}}{k}\right)^{\frac{12}{5}}\right)^{2}\left(\frac{t_{1}}{t}\right)^{\frac{3}{2}}$.

For $k \gg \frac{1}{t_{1}}$, when our calculation is valid, the second term in the last parenthesis in Eq. (4.29) is always much less than 1, because

$$
\frac{3}{8\left(t_{2} m\right)^{2}}\left(k_{c} t_{1}\right)^{\frac{12}{5}} \simeq 1.4
$$

for all values of $m$. We neglect henceforth the second term in the last parenthesis in Eq. (4.29). We have then

$$
\left.n_{r}^{(1)}\right|_{k<k_{c}}(t) \equiv \int^{k_{c}} d k \frac{d n_{r}^{(1)}}{d k}(k, t) \simeq \frac{3 C B^{2}}{2 \pi t_{1}} m t_{2}\left(\frac{t_{1}}{t}\right)^{\frac{3}{2}}
$$

for the density of axions with $k<k_{c}$ that are excited by the resonance proper. As a fraction of the density of axions in the zero mode $\left[n^{(0)}=\frac{B^{2}}{2 t_{1}}\right.$; see Eq. (2.11)] this is

$$
\frac{\left.n_{r}^{(1)}\right|_{k<k_{c}}}{n^{(0)}} \simeq \frac{3 C}{\pi} m t_{2} \simeq 0.7 \times 10^{-4}\left(\frac{m}{\mu \mathrm{eV}}\right)
$$

Fig. 3 shows a log-log plot of the spectrum of excited axions. The axions excited by the resonance proper dominate in the region $k_{*}<k<k_{c}$. The region $t_{1}^{-1}<k<k_{*}$ is dominated by axions excited by the near resonance effect, as discussed in the next subsection. For $k>k_{c}$ the density of excited axions is very small, as discussed in Sec. IV D.

\section{Near resonance excitation for $\boldsymbol{k}<\boldsymbol{k}_{\boldsymbol{c}}$}

Figure 4 shows that the frequencies $\omega_{ \pm}(k, t)$ approach $\omega(k, t)$ after the resonance proper has occurred. This produces a near resonance excitation of the axion field whose strength is estimated in this subsection. We will see that the near resonance effect is strongest at times shortly before $t_{2}$ when the axion mass is still turning on and $\omega_{ \pm}(k, t)$ are both closest to $\omega(k, t)$. Let us assume $k$ is much less than $k_{c}$ so that the time $t_{k}$ of the resonance proper occurs well before $t_{2}$. We are not so interested in $k$ near $k_{c}$ because there is little time for the near resonance effect to occur in that case. The resonance proper dominates for $k$ near $k_{c}$. We choose a time $\tilde{t}$ long after $t_{k}$ but long before $t_{2}$. The precise value of $\tilde{t}$ will be irrelevant. The contribution of the near resonance effect to the integrals $I_{ \pm}(k)$ is

$$
I_{ \pm, n r}(k)=\int_{\tilde{t}}^{\infty} d t \frac{g_{ \pm}(k, t)}{\sqrt{\omega(k, t)}} e^{i\left(h_{ \pm}(k, t)+\delta\right)} .
$$

We neglect the last two terms in the brackets on the rhs of Eq. (4.14), as we did in the previous subsection, because they are much smaller than the first term when $k \gg 1 / t_{1}$. We have then

$g_{ \pm}(k, t)=\frac{3}{8 \sqrt{t_{1} m(t)}} \frac{d m^{2}}{d T}(t) T(t)=-\frac{3}{\sqrt{t_{1}}} m(t)^{\frac{3}{2}} \Theta\left(t_{2}-t\right)$

where $\Theta(x)$ is the Heaviside step function. We approximate $\omega(k, t) \simeq m(t)$ since $k^{2} \frac{t_{1}}{t} \ll m(t)^{2}$ when $t$ approaches $t_{2}$. We have then

$$
h_{ \pm}(k, t) \simeq h_{ \pm}(k, \tilde{t}) \pm \frac{2 k}{\sqrt{3}}\left(\sqrt{t_{1} t}-\sqrt{t_{1} \tilde{t}}\right)
$$

and 


$$
\begin{aligned}
I_{ \pm, n r}(k) & \simeq-\frac{3}{\sqrt{t_{1}}} \int_{\tilde{t}}^{t_{2}} d t m(t) e^{i\left(\tilde{\delta}_{ \pm}(k) \pm \frac{2 k}{\sqrt{3}} \sqrt{t_{1} t}\right)} \\
& \simeq \pm i \frac{3 \sqrt{3} m}{t_{1} k} \sqrt{t_{2}} e^{ \pm i \frac{2 k}{\sqrt{3}} \sqrt{t_{1} t_{2}}+i \tilde{\delta}_{ \pm}(k)} \\
& \times\left[1+\mathcal{O}\left(\left(\frac{\tilde{t}}{t_{2}}\right)^{\frac{5}{2}}, \frac{1}{k \sqrt{t_{1} t_{2}}}\right)\right],
\end{aligned}
$$

where

$$
\tilde{\delta}_{ \pm}(k)=\delta+h_{ \pm}(k, \tilde{t}) \mp \frac{2 k}{\sqrt{3}} \sqrt{t_{1} \tilde{t}}
$$

We add $I_{+}(k)$ and $I_{-}(k)$ in quadrature in Eq. (4.23), i.e., we set $|I(k)|^{2}=\left|I_{+}(k)\right|^{2}+\left|I_{-}(k)\right|^{2}$, because their relative phases vary rapidly with $k$. This yields

$$
\begin{aligned}
\frac{d n_{n r}^{(1)}}{d k}(k, t) & \simeq \frac{C B^{2}}{2 \pi^{2} k}\left(\frac{3 \sqrt{3} m \sqrt{t_{2}}}{t_{1} k}\right)^{2}\left(\frac{t_{1}}{t}\right)^{\frac{3}{2}} \\
& \simeq \frac{9 C B^{2}}{2 \pi^{2} t_{1}} \frac{1}{k}\left(\frac{k_{c}}{k}\right)^{2}\left(\frac{t_{1}}{t}\right)^{\frac{3}{2}} .
\end{aligned}
$$

The density of axions produced by the near resonance effect is thus

$n_{\mathrm{nr}}^{(1)}(t)=\int_{k_{\mathrm{IR}}}^{k_{c}} d k \frac{d n_{n r}^{(1)}}{d k}(k, t) \simeq \frac{9}{4 \pi^{2}} \frac{C B^{2}}{t_{1}}\left(\frac{k_{c}}{k_{\mathrm{IR}}}\right)^{2}\left(\frac{t_{1}}{t}\right)^{\frac{3}{2}}$

where $k_{\mathrm{IR}}$ is an infrared cutoff, of order $1 / t_{1}$, below which our calculation is unreliable. We solved Eq. (4.2) numerically to validate the analytical estimates. The numerical work does not make the $k t_{1} \gg 1$ approximations made in the analytical work. The numerical results, shown as grey dots in Fig. 3, are in good qualitative agreement with the analytical results even for $k$ near $t_{1}$, implying that $k_{\mathrm{IR}} \simeq 1 / t_{1}$.

As a fraction of the axions in the zero mode, the number of axions excited by the near resonance effect is

$$
\frac{n_{n r}^{(1)}}{n^{(0)}} \simeq \frac{9 C}{2 \pi^{2}}\left(\frac{k_{c}}{k_{\mathrm{IR}}}\right)^{2} \simeq 0.06\left(\frac{1}{t_{1} k_{\mathrm{IR}}}\right)^{2}\left(\frac{m}{\mu \mathrm{eV}}\right)^{\frac{5}{3}}
$$

Since $k_{\mathrm{IR}}$ is of order $t_{1}$, the fraction of axions that get excited is of order one if the axion mass is larger than a few $\mu \mathrm{eV}$. The near resonance effect dominates for $k<k_{*}$ with

$$
k_{*}=k_{c}\left(\frac{5}{2 \pi m t_{2}}\right)^{\frac{5}{16}} \simeq \frac{k_{c}}{22}\left(\frac{\mu \mathrm{eV}}{m}\right)^{\frac{5}{16}}
$$

whereas the resonance proper dominates for $k>k_{*}$.

\section{Resonant excitation for $\boldsymbol{k}>\boldsymbol{k}_{\boldsymbol{c}}$}

We estimate here the density of axions that are excited after $t_{2}$, i.e., for $k>k_{c}$. The time at which the resonance proper occurs is given in Eq. (4.8). The same expression for $I_{+}(k)$, Eq. (4.25), applies here but with

$$
g_{+}\left(k, t_{k}\right)=-\frac{9}{8 t_{2}^{2} \sqrt{t_{1} m}}\left(\frac{k_{c}}{k}\right)^{4}
$$

and

$$
\omega\left(t_{k}\right)\left(\frac{d m}{d t}\left(t_{k}\right)+\frac{m\left(t_{k}\right)}{2 t_{k}}\right)=\left(\frac{k_{c}}{k}\right)^{2} \frac{m^{2}}{t_{2}} .
$$

Hence the axions excited by the resonance proper have spectral density

$$
\frac{d n_{r}^{(1)}}{d k}(k, t) \simeq \frac{81 C B^{2}}{64 \pi t_{1} k} \frac{1}{\left(t_{2} m\right)^{3}}\left(\frac{k_{c}}{k}\right)^{6}\left(\frac{t_{1}}{t}\right)^{\frac{3}{2}}
$$

for $k>k_{c}$, and density

$\left.n_{r}^{(1)}\right|_{k>k_{c}}(t) \equiv \int_{k_{c}}^{\infty} \frac{d n_{r}^{(1)}}{d k}(k, t) \simeq \frac{27 C B^{2}}{128 \pi t_{1}} \frac{1}{\left(t_{2} m\right)^{3}}\left(\frac{t_{1}}{t}\right)^{\frac{3}{2}}$.

As a fraction of the axions in the zero mode this is

$$
\frac{\left.n_{r}^{(1)}\right|_{k>k_{c}}}{n^{(0)}} \simeq \frac{27 C}{64 \pi} \frac{1}{\left(t_{2} m\right)^{3}}=2 \times 10^{-22}\left(\frac{\mu \mathrm{eV}}{m}\right)^{3}
$$

which is always very small. For most plausible values of $H_{I}$, it is much smaller than the contribution from the quantum mechanical fluctuations in the axion field, discussed in Sec. III. The reason why resonant excitation is such a small effect for $k>k_{c}$ is that the axion mass is constant after $t_{2}$ and hence the term $T \frac{d m^{2}}{d T}$ is absent from $g_{+}(k, t)$. For $k>k_{c}$ the contribution from the near resonance effect is found to be even smaller than the small contribution from the resonance proper.

\section{SUMMARY}

We derived the spectrum of cold axions after the QCD phase transition has been completed for wave vectors $k$ at time $t_{1}$ much larger than $1 / t_{1} . t_{1}$ was defined in Eq. (2.5). Two kinds of axion field perturbations contribute to the spectrum in case inflation occurs after the PQ phase transition. First, there are the perturbations that originate as quantum mechanical fluctuations in the axion field during the inflationary phase. Those were treated in Sec. III. Their contribution to the cold axion energy density is very small when the isocurvature constraints from the $\mathrm{CMB}$ anisotropy observations are taken into account. 
Second, there are the fluctuations induced in the axion field by the adiabatic perturbations in the primordial plasma. These were treated in Sec. IV. We show that the adiabatic perturbations in the plasma resonantly pump low momentum axions into modes of wave vector magnitude up to of order $\sqrt{3} m$ where $m$ is the zero temperature axion mass. The process conserves axion number and transfers energy from the primordial plasma to the axion fluid. The spectrum of axions that are resonantly excited is given in Eqs. (4.29), (4.38), and (4.44), and illustrated in Fig. 3. The fraction of cold axions that get excited is of order one if the axion mass is larger than a few $\mu \mathrm{eV}$.

The process of axion field excitation occurs in qualitatively the same way whether inflation occurs before or after the PQ phase transition since the initial cold axion momenta are in either case of order $1 / t_{1}$ or less, much smaller than the momenta to which they get resonantly excited.
Resonant excitation of the axion fluid during the QCD phase transition does not change the status of the axion as a cold dark matter candidate since the excited axions are nonrelativistic again long before the onset of large scale structure formation. However, the excitation spectrum may have implications for observation. In future work, we intend to study its implications for the formation of axion miniclusters [15] and for the cosmic time at which cold axion dark matter first Bose-Einstein condenses by gravitational self-interactions [16].

\section{ACKNOWLEDGMENTS}

P. S. thanks Richard Woodard for useful discussions. This work was supported in part by the U.S. Department of Energy under Grant No. DE-SC0022148 at the University of Florida.
[1] R. D. Peccei and H. Quinn, Phys. Rev. Lett. 38, 1440 (1977); Phys. Rev. D 16, 1791 (1977).

[2] S. Weinberg, Phys. Rev. Lett. 40, 223 (1978); F. Wilczek, Phys. Rev. Lett. 40, 279 (1978).

[3] J. Kim, Phys. Rev. Lett. 43, 103 (1979); M. A. Shifman, A. I. Vainshtein, and V. I. Zakharov, Nucl. Phys. B166, 493 (1980); M. Dine, W. Fischler, and M. Srednicki, Phys. Lett. 104B, 199 (1981); A. Zhitnitskii, Sov. J. Nucl. 31, 260 (1980).

[4] J. Preskill, M. Wise, and F. Wilczek, Phys. Lett. 120B, 127 (1983); L. Abbott and P. Sikivie, Phys. Lett. 120B, 133 (1983); M. Dine and W. Fischler, Phys. Lett. 120B, 137 (1983).

[5] Reviews of axion cosmology include: P. Sikivie, Lect. Notes Phys. 741, 083513 (2005); D. J. E. Marsh, Phys. Rep. 643, 1 (2016).

[6] D. J. Gross, R. D. Pisarski, and L. G. Yaffe, Rev. Mod. Phys. 53, 43 (1981).

[7] Sz. Borsanyi et al., Nature (London) 539, 69 (2016).

[8] C. Bonati et al., EPJ Web Conf. 137, 08004 (2017).

[9] A. A. Starobinsky, Phys. Lett. 91B, 99 (1980); D. Kazanas, Astrophys. J. 241, L59 (1980); K. Sato, Mon. Not. R. Astron. Soc. 195, 467 (1981).
[10] A. Guth, Phys. Rev. D 23, 347 (1981); A. D. Linde, Phys. Lett. 108B, 389 (1982); A. Albrecht and P. Steinhardt, Phys. Rev. Lett. 48, 1220 (1982).

[11] M. Axenides, R. H. Brandenberger, and M. S. Turner, Phys. Lett. 126, 178 (1983); P. J. Steinhardt and M. S. Turner, Phys. Lett. 129B, 51 (1983); A. D. Linde, Phys. Lett. 158B, 375 (1985); D. Seckel and M. S. Turner, Phys. Rev. D 32, 3178 (1985); D. H. Lyth, Phys. Lett. B 236, 408 (1990); M. S. Turner and F. Wilczek, Phys. Rev. Lett. 66, 5 (1991).

[12] V. F. Mukhanov and G. V. Chibisov, JETP Lett. 33, 532 (1981); S. W. Hawking, Phys. Lett. 115B, 295 (1982); A. Guth and S.-Y. Pi, Phys. Rev. Lett. 49, 1110 (1982); J. M. Bardeen, P. J. Steinhardt, and M. S. Turner, Phys. Rev. D 28, 679 (1983).

[13] L. Husdal, Galaxies 4, 78 (2016).

[14] S. Dodelson, Modern Cosmology (Academic Press, New York, 2003).

[15] E. W. Kolb and I. I. Tkachev, Phys. Rev. Lett. 71, 3051 (1993).

[16] P. Sikivie and Q. Yang, Phys. Rev. Lett. 103, 111301 (2009); O. Erken, P. Sikivie, H. Tam, and Q. Yang, Phys. Rev. D 85, 063520 (2012). 